\title{
High-pressure processing of milk alleviates atopic dermatitis in DNCB-induced Balb/c mice
}

\author{
Jieun Lee ${ }^{1}$ - Eun-Ji Choi ${ }^{1}$ - Sun Young Park ${ }^{2}$. \\ Ga Young Jeon ${ }^{3} \cdot$ Ja-Young Jang ${ }^{1}$. \\ Young Jun $\mathrm{Oh}^{1}$ • Seul Ki Lim ${ }^{1} \cdot$ Min-Sung Kwon ${ }^{1}$ • \\ Tae-Woon Kim ${ }^{2}$ • Jong-Hee Lee ${ }^{2}$. \\ Hae Woong Park ${ }^{2}$ • Hyun Ju Kim ${ }^{1}$. \\ Jong Soo Kang ${ }^{5}$. Jung Tae Jeon ${ }^{4}$ - Hak-Jong Choi ${ }^{1}$
}

Received: 5 November 2014 / Revised: 7 May 2015 / Accepted: 22 May 2015 /

Published online: 23 June 2015

(C) INRA and Springer-Verlag France 2015

\begin{abstract}
High pressure (HP) is recognized as the alternative process technology of heat treatment in food production. Although milk was the first food product to be treated with HP, the allergenicity of HP-treated milk remains to be elucidated. Thus, this study was conducted to investigate the effects of HP treatment on the alleviation of atopic dermatitis (AD) caused by milk in a mouse model. To investigate the effect of $\mathrm{HP}$ treatment on the alleviation of $\mathrm{AD}, \mathrm{Balb} / \mathrm{c}$ mice were fed HP-treated milk orally for 7 days following induction of AD with 1-chloro-2,4-dinitro-benzene (DNCB). Mice fed with HP-treated milk exhibited markedly lower serum IgE level than DNCB-treated mice. These results suggest that HP may be applicable as an alternative process for the development of hypoallergenic milk products.
\end{abstract}

Keywords High pressure $\cdot$ Milk $\cdot$ Atopic dermatitis

Hak-Jong Choi

hjchoi@wikim.re.kr

1 Metabolism and Functionality Research Group, World Institute of Kimchi, Gwangju 503-360, Korea

2 Microbiology and Fermentation Research Group, World Institute of Kimchi, Gwangju 503-360, Korea

3 Food Science and Nutrition, Yonsei University, Seoul 120-749, Korea

4 Food Science and Nutrition, Pusan National University, Busan 609-735, Korea

5 Ajou University, Suwon 443-749, Korea 


\section{Introduction}

Atopic dermatitis (AD) is a common public health problem worldwide that is associated with social and financial burdens, with a lifetime prevalence of $1-3 \%$ in adults and $20 \%$ in children (Leung 2000; DaVeiga 2012). AD patients with primary $\mathrm{T}$ cell immunodeficiency disorders frequently have elevated serum immunoglobulin (Ig) $\mathrm{E}$ levels, eosinophilia, and eczematoid skin lesions indistinguishable from AD (Leung 1999). Because T helper (Th) type 1 cells cross-regulate Th2 cells in some systems, allergen-specific Th1 cells have been assumed to regulate allergic disease and asthma (Umetsu and DeKruyff 2006). Th1 cells inhibit the development and proliferation of Th2 cells, and IgE production is reciprocally regulated by interleukin (IL)-4 and interferon- $\gamma($ IFN- $\gamma)$, suggesting that protection from allergy is due to the development of inhibitory allergen-specific Th1 cells (Umetsu and DeKruyff 2006). Therefore, enhancement of Th1-type immunity and suppression of Th2-type immunity might be an effective therapy for the management of AD (Won et al. 2011).

Milk-induced allergic disorders result from immunologic pathways that include the activation of effector cells through food-specific IgE antibodies and cell-mediated reactions resulting in subacute or chronic inflammation, or a combination of the above (NIAID-Sponsored Expert Panel et al. 2010; Kaczmarski et al. 2013). Cow's milk proteins represent the first source of antigens encountered in large quantities in infancy (Isolauri and Turjanmaa 1996; $\mathrm{Bu}$ et al. 2013). Among these antigens, $\beta$-lactoglobulin $(\beta-\lg ), \alpha$ lactalbumin $(\alpha$-la), and caseins are the main allergens in cow's milk. Finding new and effective processing technologies to reduce the allergen content of milk is therefore important for controlling milk allergies (Bu et al. 2013). Several studies from different countries have shown that the consumption of unpasteurized milk has protective effects against atopic sensitization, hay fever, and asthma, although the evidence is inconsistent (Sozańska et al. 2013; Lluis et al. 2014). The reasons for these possible protective effects are unclear. Milk treatments, such as heating and homogenization, can affect heat-sensitive components or the physical structure of milk fat and abrogate the protective effect of unpasteurized milk against allergy (von Mutius and Vercelli 2010; Braun-Fahrländer and von Mutius 2011). Heat sterilization of milk causes the aggregation of whey proteins such as $\beta$-lg and $\alpha$-la, and pasteurized $\beta$-lg enhances levels of $\operatorname{IgE}$ as well as Th2 cytokine responses in the initial sensitization step (Roth-Walter et al. 2008).

High-pressure processing (HPP) is a relatively novel processing technique in the food industry. HPP can cause structural changes in milk proteins, such as denaturation and formation of aggregates (Iametti et al. 1997), and these changes may also influence the allergenic potential of milk proteins $(\mathrm{Bu}$ et al. 2013). High-pressure treatment enhances the antigenicity of $\beta-\lg$ in whey protein isolate solution products, sweet whey, and raw skim milk (Kleber et al. 2007). The increase in antigenicity may be associated with exposure of epitopes that are hidden in the native protein conformation, as pressure-induced unfolding and aggregation make them accessible to antibodies (Kleber et al. 2007). In addition, high-pressure treatment during enzymatic hydrolysis can effectively reduce the antigenicity and serum $\operatorname{IgE}$ binding properties of milk protein hydrolysates (Beran et al. 2009; Bu et al. 2013). As milk and its products are mainly consumed in pasteurized forms, we compared the allergenicity of heat-treated milk and HPP-treated milk using a 1-chloro-2,4-dinitro-benzene (DNCB)-induced AD model in $\mathrm{Balb} / \mathrm{c}$ mice. 


\section{Materials and methods}

\subsection{Animals and treatment}

Six-week-old female Balb/c mice were purchased from Damul Science (Daejeon, Korea) and provided with a rodent diet and water ad libitum in a temperature- and humidity-controlled pathogen-free animal facility at the World Institute of Kimchi, Korea. After the 7-day adaptation period, mice were assigned to one of six groups $(n=5$ each): a non-induction group (NI group, mice treated with vehicle); a negative control group (NC group, mice sensitized with $\mathrm{DNCB}$ and fed water); a positive control group (PC group, mice sensitized with DNCB and fed the anti-histamine agent kerotifen at $1 \mathrm{mg} . \mathrm{kg}^{-1}$; an HPP milk-fed group (HPP group, mice sensitized with DNCB and fed HPP-treated milk); a low temperature-long time (LTLT) pasteurized milk-fed group (LTLT group, mice sensitized with DNCB and fed LTLT milk); and an ultra-high temperature (UHT) pasteurized milk-fed group (UHT group, mice sensitized with DNCB and fed UHT milk). The backs of the mice were shaved with electric clippers before sensitization. For the sensitization process (Fig. 1), $0.2 \mathrm{~mL}$ of $1 \%$ DNCB in acetone/olive oil (3:1) was applied to the dorsal skin of the animals for 2 days, on day 0 and day 3. On day 7 and day $10,0.2 \mathrm{~mL}$ of $0.2 \%$ DNCB was applied for prior sensitization. The mice were fed each fluid (water, kerotifen, HPP milk, LTLT milk, or UHT milk) for 7 days.

\subsection{Evaluation of dermatitis}

The severity of the dermatitis in the dorsal skin of DNCB-induced AD mice was evaluated on day 18. The severity of four symptoms (erythema/hemorrhage, edema, excoriation/erosion, and scarring/dryness) was scored as 0 (none), 1 (mild), 2 (moderate), or 3 (severe). Dermatitis score was defined as the sum of these individual scores in accordance with the method of Matsuda et al. (1997).

\subsection{Histological analysis}

The dorsal skins were fixed with $10 \%$ formaldehyde and embedded in paraffin, and thin sections were made. The skin sections were stained with hematoxylin and eosin (H\&E) for evaluation of edema. The other sections were stained with toluidine blue or Congo red for detection of mast cells or eosinophils, respectively. Images were

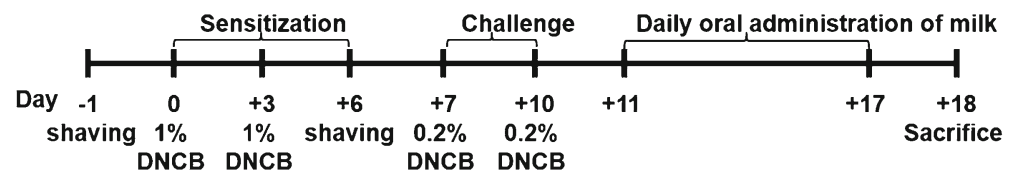

Fig. 1 Schematic diagram of the study protocol. Mice were divided into six groups ( $n=5$ per group). To induce AD-like immunologic and skin lesions, DNCB was applied to the dorsal skin. Shaved dorsal regions of all the mice were sensitized epicutaneously with $0.2 \mathrm{~mL}$ of $1 \% \mathrm{DNCB}$ on day 0 and day 3 . After sensitization, $0.2 \mathrm{~mL}$ of $0.2 \%$ DNCB was used to challenge the animals as the previous sensitization on day 7 and day 10 . After the challenge, mice were orally administrated $0.2 \mathrm{~mL}$ of deionized water, kerotifen $\left(1 \mathrm{mg}^{\mathrm{kg}}{ }^{-1}\right), \mathrm{HPP}$, LTLT, and UHT milk, seven times for 1 week. Animals were sacrificed for 18 days after the first application of $\mathrm{DNCB}$ 
captured using an Olympus DP 73 (Olympus Optical Co., Hamburg, Germany) and viewed at a magnification of 100 .

\subsection{Measurement of total IgE and IgG2a in serum}

Blood samples were collected from the mice after sacrifice, and serum samples were obtained by centrifugation $(3000 \times g, 10 \mathrm{~min})$. Serum cytokine levels were measured using ELISA OptEIA Mouse Sets (BD Biosciences, San Jose, CA, USA) for IgE and IgG2a according to the manufacturer's instructions.

\subsection{Cell population in inguinal lymph nodes}

On day 18 , the experimental mice were sacrificed and their inguinal lymph nodes were removed. Images of the lymph nodes were captured and their size was measured. For cell counts, the inguinal lymph nodes were homogenized, and the cell suspension was stained with $0.4 \%$ trypan blue and placed in a hemocytometer under a microscope. For determination of $\mathrm{T}$ or $\mathrm{B}$ cell numbers, the cell suspension was incubated with FITC-labeled anti-CD3 $\varepsilon$ or anti-CD19 monoclonal antibodies on ice for $30 \mathrm{~min}$. The expression of surface molecules was analyzed by flow cytometry. Flow cytometry was performed using a FACSCanto II (BD Biosciences), and data analysis was performed using FlowJo software (Treestar, Inc., San Carlos, CA, USA).

\subsection{Measurement of cytokines in lymph node cell culture solution}

Inguinal lymph node cells $\left(5 \times 10^{5}\right.$ cells $)$ were stimulated with immobilized anti-CD3 $\mathrm{mAb}(1 \mu \mathrm{g})$ for $48 \mathrm{~h}$ at $37{ }^{\circ} \mathrm{C}$ under $5 \% \mathrm{CO}_{2}$ in an incubator. After incubation, the culture supernatants were collected and stored at $-80{ }^{\circ} \mathrm{C}$ for later analysis. Multiple soluble cytokines (IL-10, IL-17A, TNF, IFN- $\gamma$, IL-6, IL-4, and IL-2) were measured by flow cytometry using a Cytometric Bead Assay (CBA) mouse Th1/Th2/Th17 kit (BD Biosciences) according to the manufacturer's instructions.

\subsection{Statistical analysis}

All data collected in this study were analyzed using SPSS 19.0 (SPSS Inc., Chicago, IL, USA). Statistical comparisons between means were carried out using one-way ANOVA followed by Duncan's test. A $p$ value $<0.05$ was considered statistically significant. Pairwise significance test was done using NC or PC group as control groups.

\section{Results}

\subsection{Effect of HPP milk on DNCB-induced AD-like skin lesions in mice}

To examine the effect of HPP milk on the alleviation of AD using a mouse model, DNCB was repeatedly applied twice per week to the dorsal skin of Balb/c mice. 
Figure 2a, $\mathrm{b}$ shows the clinical features and dermatitis scores of Balb/c mice treated with DNCB. The Balb/c mice developed AD-like skin lesions following repeated application of DNCB, as indicated by their skin lesion scores, whereas non-induced mice did not exhibit any skin lesions. The AD induced by repeated application of DNCB produced all four dermatitis symptoms of erythema/hemorrhage, edema, excoriation/erosion, and scarring/dryness of the dorsal skin. These symptoms of AD were reduced in the HPP milk and kerotifen treatment groups compared with the NC group $(p<0.05)$. The LTLT- and UHT-milk-fed groups showed weaker edema and excoriation than the NC group $(p<0.05)$, whereas the LTLT- and UHT-milk-fed groups only exhibited stronger AD symptoms than the PC group $(p<0.05)$. These results indicate that HPP milk feeding alleviates $\mathrm{AD}$ symptoms in mice.

\subsection{Effect of HPP milk on the serum level of IgE and IgG2a}

The hyperproduction of $\operatorname{IgE}$ is a characteristic feature of $\mathrm{AD}$ and is mediated by $\mathrm{Th} 2$ response. On the other hand, the production of $\mathrm{IgG} 2 \mathrm{a}$ is modulated by Th1 response (Corry and Kheradmand 1999; Leung 1999). As shown in Fig. 3a, the total IgE level of the NC group was higher than that of the NI group, whereas kerotifen- and HPP-milkfed mice showed significantly lower serum levels of IgE than the NC group, LTLT, and UHT milk groups. Serum IgG2a levels of the NC, LTLT, and UHT groups were similar to that of the NI group, whereas the PC and HPP groups exhibited significantly higher levels of IgG2a than the NC, LTLT, and UHT milk groups (Fig. 3b). The IgE/IgG2a ratio in mice supplemented with kerotifen and fed HPP milk was significantly lower than that of the DNCB-treated group without milk supplementation; this ratio was significantly higher in the mice fed UHT and LTLT milk. The ratio was significantly lower in the PC and HPP groups than in the NC group, even though they were treated with DNCB for AD induction (Fig. 3c).
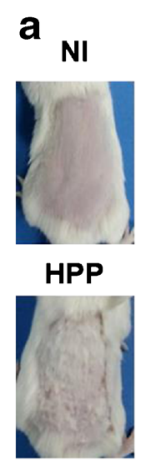
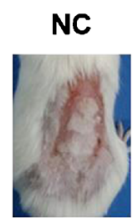

LTLT

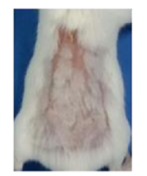

PC

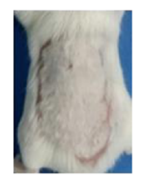

UHT

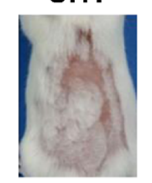

b

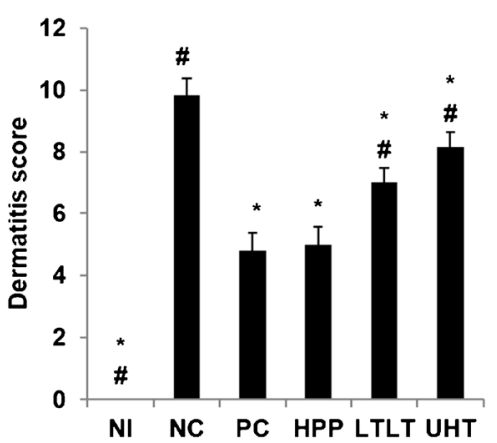

Fig. 2 Effects of HPP milk on atopic dermatitis symptoms. a Aspects of DNCB-induced dermatitis in Balb/c mice were taken on day 18. b Dermatitis scores were defined as the sum of scores for four symptoms: erythema/hemorrhage, edema, excoriation/erosion, and scarring/dryness. The results are expressed as means \pm SE. Asterisk indicates significant difference compared to the NC group $(p<0.05)$; number sign indicates significant difference compared to the PC group $(p<0.05)$. NI non-induction group, NC DNCB-only group, $P C \mathrm{DNCB}+$ kerotifen group, HPP DNCB + HPP milk group, LTLT DNCB + LTLT milk group, UHT DNCB + UHT milk group 

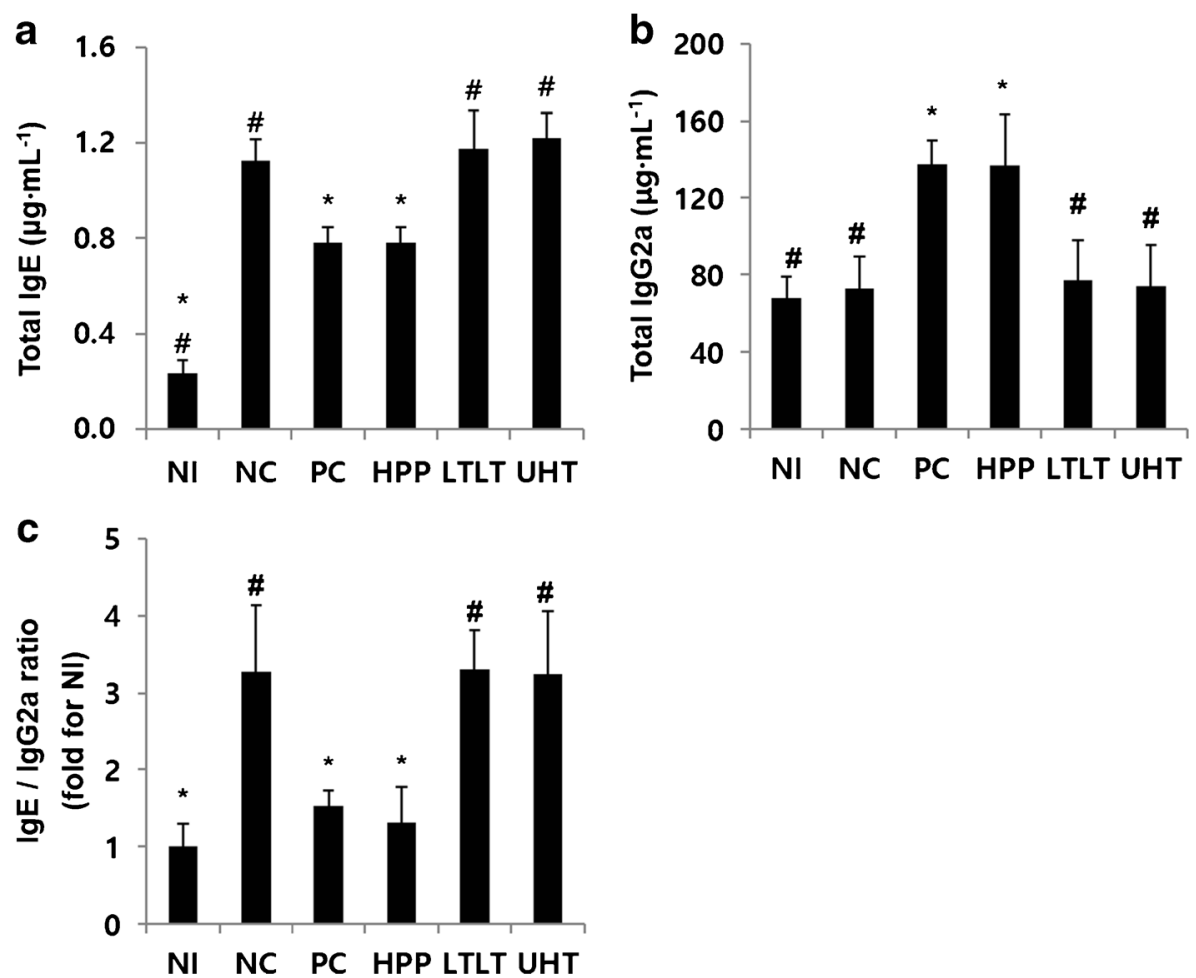

Fig. 3 Effect of milk on the level of serum antibodies. The concentration of a total IgE and $\mathbf{b}$ total IgG2a in collected serum was determined by ELISA. The ratio of $\mathbf{c} \mathrm{IgE} / \mathrm{IgG} 2 \mathrm{a}$ was converted into fold for NI. Data are shown as mean \pm SE. Asterisk indicates significant difference compared to the NC group $(p<0.05)$; number sign indicates significant difference compared to the PC group $(p<0.05)$. NI non-induction group, NC DNCBonly group, $P C$ DNCB + kerotifen group, HPP DNCB + HPP milk group, LTLT DNCB + LTLT milk group, $U H T$ DNCB + UHT milk group

\subsection{HPP decreases skin thickness and local infiltration of mast cells and eosinophils in mice}

Mast cells and eosinophils infiltrate local sites and mainly influence the induction of AD. Figure 4a, b shows that DNCB treatment significantly increased skin thickness in $\mathrm{Balb} / \mathrm{c}$ mice. However, treatment with kerotifen, HPP, and LTLT milk inhibited DNCBinduced $\mathrm{AD}$ skin lesions. As shown in Fig. $4 \mathrm{c}-\mathrm{f}$, accumulation of mast cells and eosinophils was increased in DNCB-induced mice $(p<0.05)$. However, oral administration of kerotifen, HPP, and LTLT milk significantly suppressed the accumulation of the cells in dorsal inflammatory sites compared with the NC group. Histological analysis by toluidine blue and Congo red staining revealed that UHT milk did not inhibit the infiltration of mast cells or eosinophil accumulation in the skin lesions.

\subsection{HPP reduces the size and cell number of lymph nodes in mice}

During the adaptive immune response, a lymphoid organ near the site of local inflammation generally takes charge of antigen presentation, lymphocyte differentiation, and proliferation for rapid and effective elimination of antigens. Accordingly, 
a
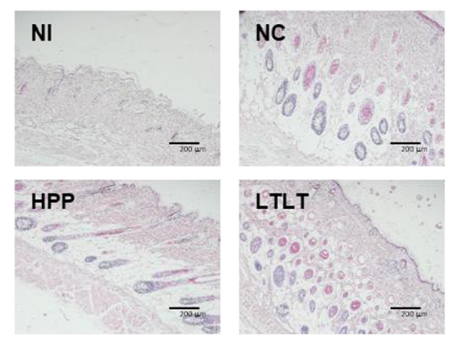

C
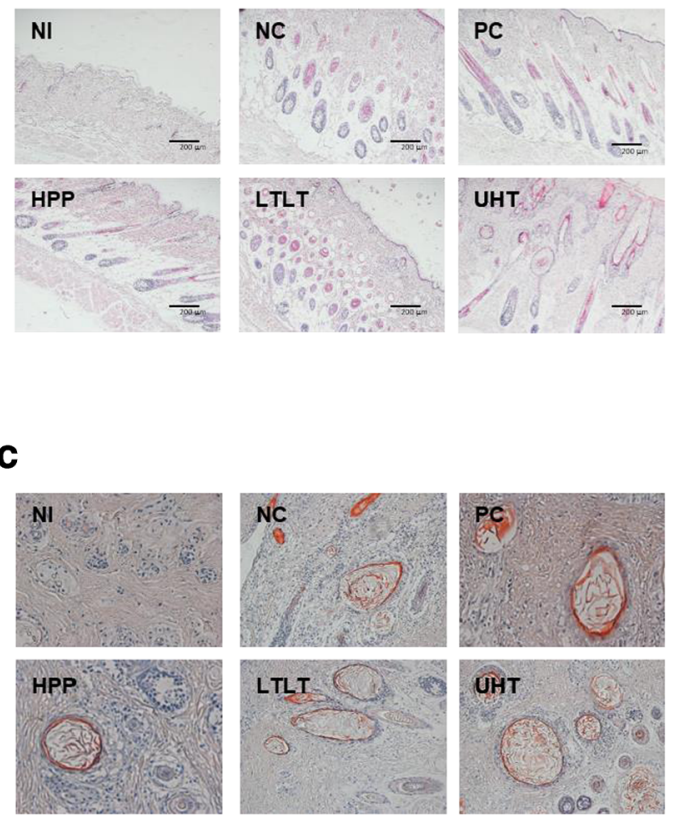

b

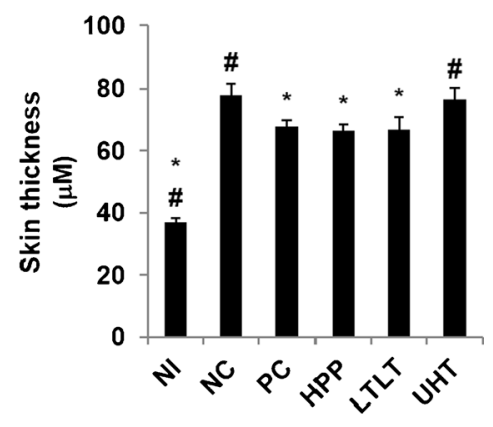

d

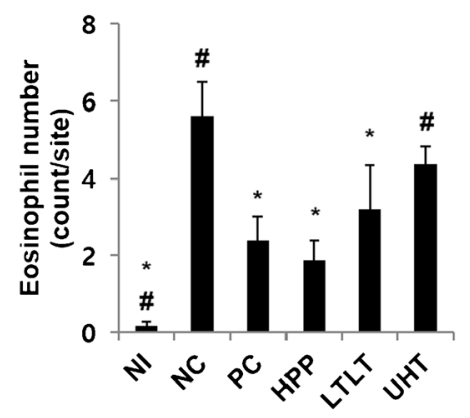

f
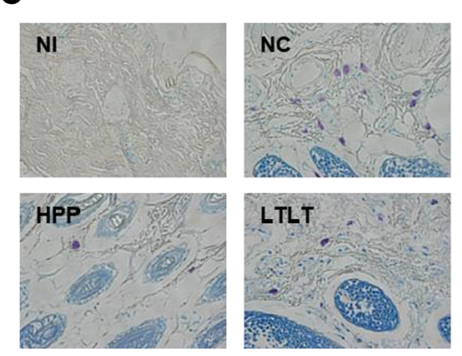
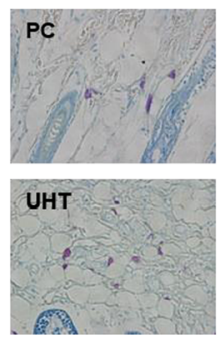

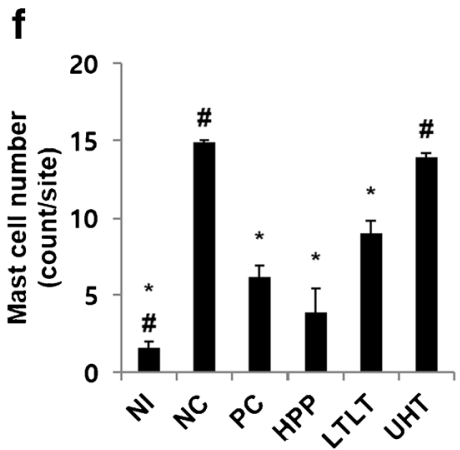

Fig. 4 Status of Balb/c mouse dorsal skin treated with DNCB repeatedly. The dorsal skins were removed, fixed with $10 \%$ formaldehyde solution, and embedded in paraffin. The skin sections were stained with hematoxylin and eosin (a, b), Congo Red (c, d), and toluidine blue (e, f). Data are represented as means \pm SE. Asterisk indicates significant difference compared to the NC group $(p<0.05)$; number sign indicates significant difference compared to the PC group $(p<0.05)$. NI non-induction group, NC DNCB-only group, $P C$ DNCB + kerotifen group, HPP DNCB + HPP milk group, LTLT DNCB + LTLT milk group, UHT DNCB + UHT milk group

lymph nodes are enlarged, and their cell number is increased (Won et al. 2011). As shown in Fig. 5, the size of the inguinal lymph nodes was enlarged, and the total number of lymph node cells in DNCB-treated mice was also increased in the case of $\mathrm{AD}$ as compared with the NI group. Oral administration of HPP lowered total cell, T cell, and B cell number and suppressed lymph node enlargement, compared with the 

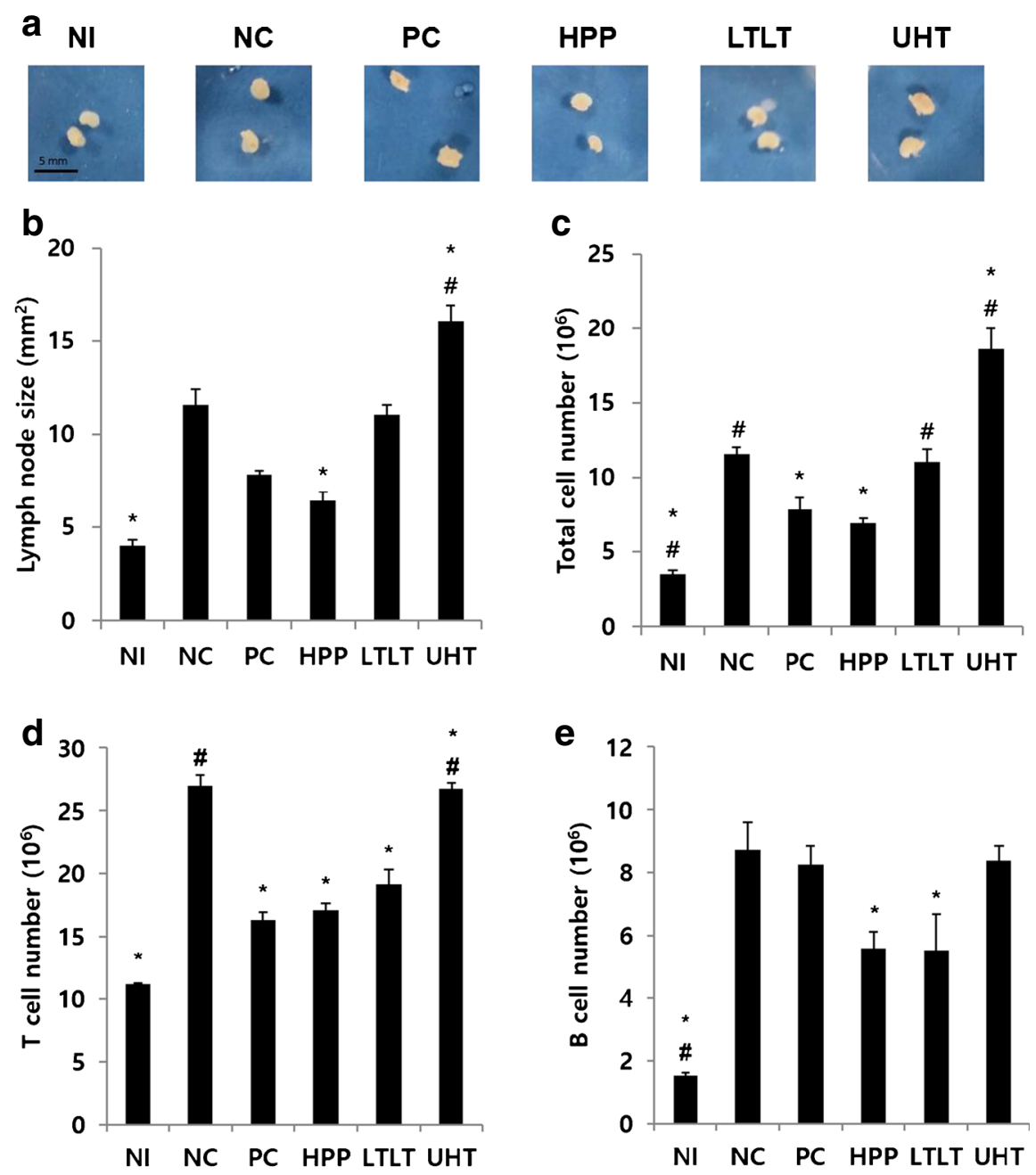

T cell gate
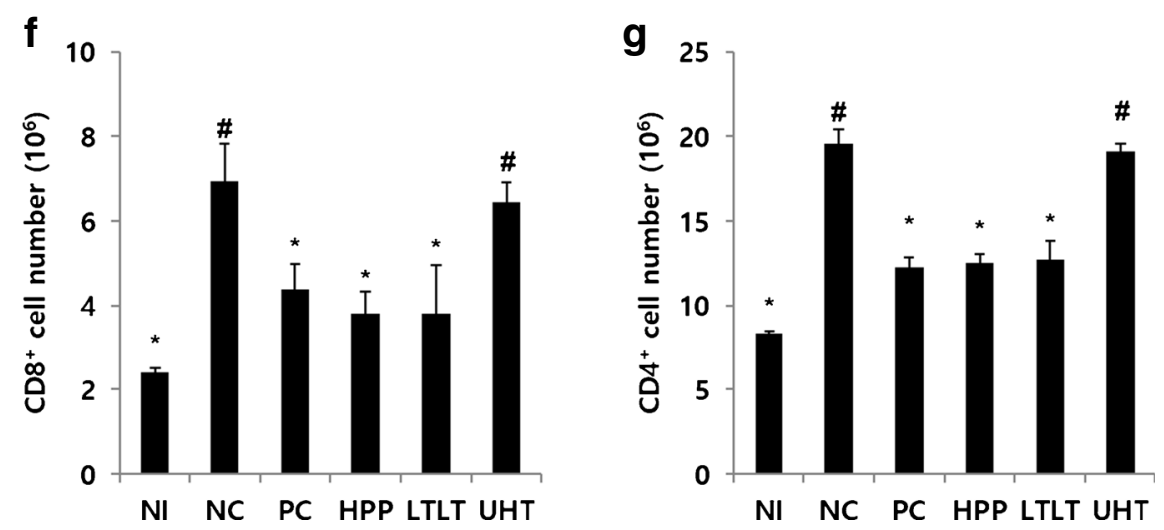
1 Fig. 5 Effect of the ingestion of milk on the drain lymph nodes. a The drain lymph nodes on day 18. b The size of lymph nodes was measured on day 18. c Total numbers of lymph node cells. Absolute numbers of $\mathrm{T}$ cells (d) and B cells (e) based on CD3e and CD19 expression. f Absolute numbers of CD8 ${ }^{+}$T cells and CD4 ${ }^{+}$ T cells. Cell suspensions were obtained from excised lymph nodes and counted for measurement of total cell number. Then, the cells were incubated with anti-CD3 $\varepsilon$ antibody or anti-CD19 antibody for the detection of T cells and B cells, respectively, and analyzed by flow cytometry. Data are expressed as the means \pm SE. Asterisk indicates significant difference compared to the NC group $(p<0.05)$; number sign indicates significant difference compared to the PC group $(p<0.05)$. NI non-induction group, NC DNCB-only group, $P C$ DNCB + kerotifen group, HPP DNCB + HPP milk group, LTLT DNCB + LTLT milk group, $U H T$ DNCB + UHT milk group

NC group $(p<0.05)$. However, lymph node cell number and size were higher in mice fed UHT milk than in control mice $(p<0.05)$.

\subsection{HPP decreases the cytokine production of $\mathrm{T}$ cells in lymph node cells}

To address whether HPP-treated milk affects the Th1/Th2 balance at the site of inflammation, cytokine production by lymph node cells was measured. Figure $6 \mathrm{a}$ shows that the PC and HPP groups had increased production of IFN- $\gamma$, a representative Th1-type cytokine. Because IFN- $\gamma$ generally suppresses $\mathrm{T}$ cell polarization into Th2 cells that produce IL-4 and IL-5, ingestion of a potent IFN- $\gamma$ inducer such as kerotifen or HPP milk mildly decreased the secretion of IL-4 cytokines in inguinal lymph node cells; however, LTLT and UHT milk also slightly increased the production of IFN- $\gamma$, although it increased the secretion of IL-4 compared with the NC group (Fig. 6b). Cells from mice fed HPP milk showed significantly increased TNF production in culture (Fig. 6c). Secretion of IL-10 was enhanced by treatment with UHT milk, compared with the PC group (Fig. 6d, $p<0.05$ ). The IL-2, IL-6, and IL-17A cytokines did not differ significantly among DNCB-induced AD mice (Fig. 6e-g).

\section{Discussion}

In this study, we addressed the immunological activity of HPP milk to alleviate AD in mice. Our results showed that mice fed with HPP-treated milk exhibited a significantly reduced serum IgE level and AD symptoms compared with DNCB-treated mice, suggesting that HPP treatment may reduce the allergenicity of milk proteins. However, there may be a possibility that "helpful" components in the milk are not destroyed by HPP treatment. The HPP milk inhibited clinical signs of DNCB-induced AD lesions, such as redness, bleeding, eruption, and scaling, and decreased serum IgE levels and inflammatory skin thickening. Because the dermal thickness of mice fed HPP milk was lower than that of NC mice, it is possible that infiltration of immune cells such as mast cells and eosinophils was reduced in these mice. In addition, the volume of the inguinal lymph nodes, which are responsible for dorsal skin inflammation, was also reduced by oral administration of HPP milk in DNCB-induced AD mice. These results suggest that oral uptake of HPP milk inhibits the immune response of T and/or B cells, major components of lymph nodes, against DNCB invasion, thus lowering the induced immunological reaction by diminishing the recruitment of major effector cells such as mast cells and eosinophils in atopic hypersensitivity. Furthermore, the decline of serum IgE concentrations induced by HPP suppresses the activation of mast cells and eosinophils, because 

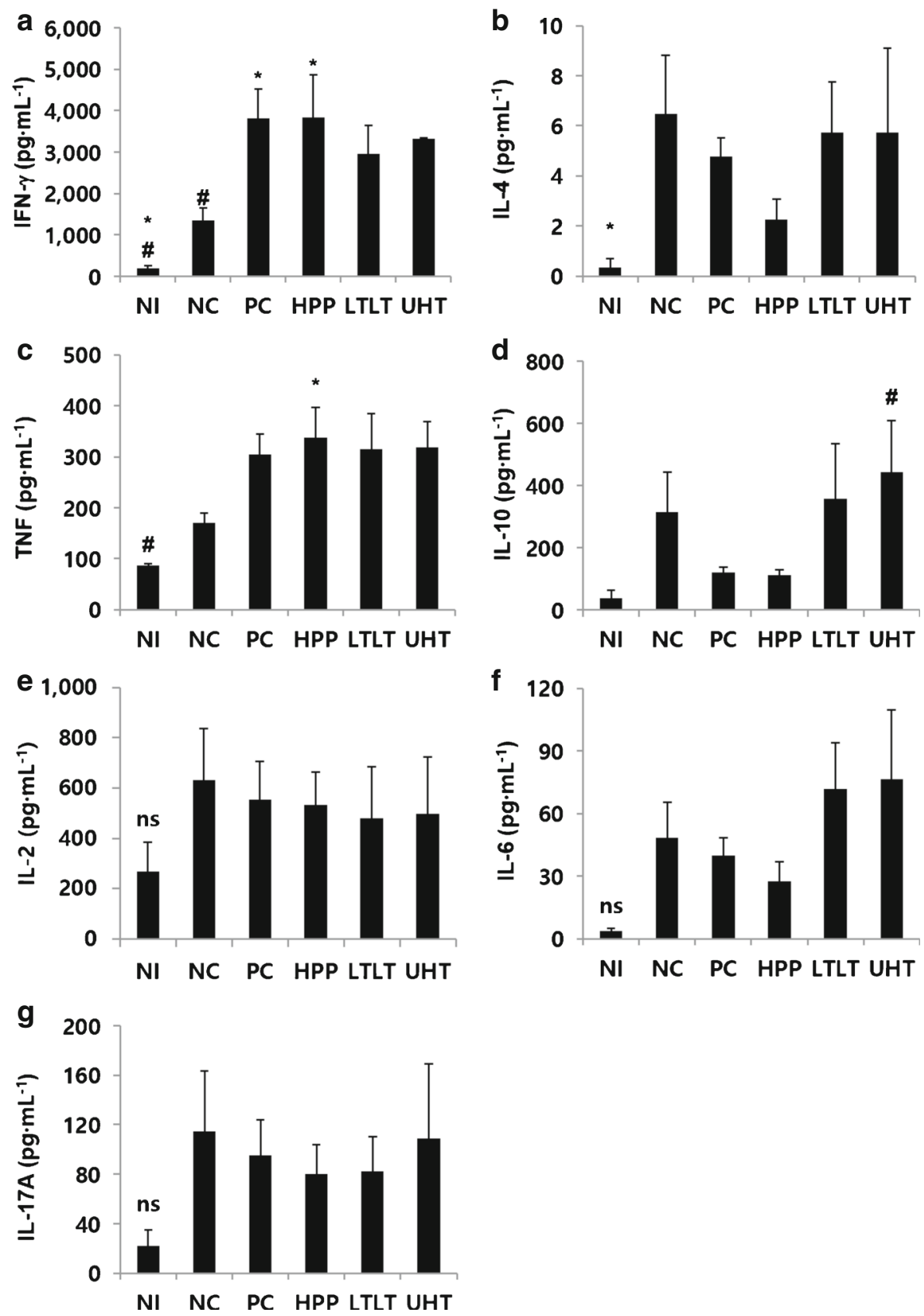

Fig. 6 Effect of the ingestion of milk on the production of a IFN- $\gamma$, b IL-4, c TNF, d IL-10, e IL-2, f IL-6, and g IL-17A in inguinal lymph nodes. Data are expressed as the means \pm SE. Asterisk indicates significant difference compared to the NC group $(p<0.05)$; number sign indicates significant difference compared to the PC group $(p<0.05)$. NI non-induction group, $N C$ DNCB-only group, $P C D N C B+$ kerotifen group, $H P P$ DNCB + HPP milk group, LTLT DNCB + LTLT milk group, UHT DNCB + UHT milk group 
they express high-affinity surface receptors for $\operatorname{IgE}$ that induce their activation as well as degranulation of inflammatory mediators such as histamine and tryptase.

We investigated whether oral administration of HPP milk influenced the balance of Th1/Th2 immune responses. IFN- $\gamma$, secreted by Th1 cells, induces cell-mediated and inflammatory immune responses and suppresses polarization immunity towards the response (Kang et al. 2009). In the present study, IFN- $\gamma$ production was elevated in inguinal lymph node cells isolated from mice fed HPP milk, whereas the concentrations of IL-4 and IL-10, which are Th2-type cytokines, were diminished. Hence, the ingestion of milk treated with HPP may lead to induction of adaptive immunity towards Th1 responses in Th2-dominant atopic Balb/c mice through the activation of IL-12-producing macrophages and restore the skewed Th1/Th2 balance to its normal condition (Kang et al. 2009). The diminished Th2 response in AD mice fed HPP milk might be able to decline to switch antibody isotype from $\operatorname{IgM}$ to $\operatorname{IgE}$ produced by B cells and to recruit eosinophils or mast cells to inflamed tissues. The balance between Th1 and Th2 responses is important for appropriate immune function, and disruption of the Th1/Th2 balance leads to various immune-related diseases such as autoimmune diseases and allergic diseases (Abramovits 2005). In allergic asthma, the number of Th 2 cells and Th 2 cytokines is increased, whereas the number of Th1 cells and Th1 cytokines is decreased (Packard and Khan 2003). Likewise, because of the beneficial effects of HPP milk on DNCB-induced dermatitis lesions and serum IgE levels, HPP milk is considered to be an enhancer of Th1 or a suppressor of Th2 responses, similar to kerotifen. However, oral intake of UHT and LTLT milk increased the production of both Th1- and Th2-type cytokines in inguinal lymph node cells, which is probably due to the antigenicity of heat-treated milk proteins ( $\mathrm{Bu}$ et al. 2013). In particular, UHT milk enhanced the production of IL-10, which is normally overexpressed in the skin of $\mathrm{AD}$ patients and is known to be related to the upregulation of humoral responses and the downregulation of Th1 responses (Ohmen et al. 1995).

In conclusion, the data presented here suggest that HPP milk may ameliorate allergen-induced skin inflammation by downregulating IgE and regulating Th1/Th2 cytokine levels. Further studies focusing on reducing the allergenicity of milk protein in comparison with unprocessed milk, HPP milk, and heat-treated milk would provide new insight into the application of HPP to milk as an alternative process for the development of hypoallergenic milk products. Therefore, human tests should be carried out to further evaluate the potential of HPP milk.

Conflict of interest The authors declare that they have no competing interests.

Compliance with Ethical Standards All applicable institutional and/or national guidelines for the care and use of laboratory animals were followed.

\section{References}

Abramovits W (2005) Atopic dermatitis. J Am Acad Dermatol 53:S86-S93

Beran M, Klubal R, Molik P, Strohalm J, Urban M, Klaudyova AA, Prajzlerova K (2009) Influence of highhydrostatic pressure on tryptic and chymotryptic hydrolysis of milk proteins. High Press Res 29:23-27

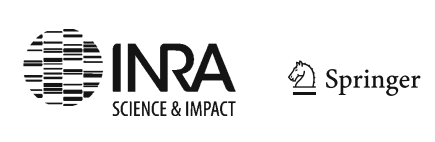


Braun-Fahrländer C, von Mutius E (2011) Can farm milk consumption prevent allergic diseases? Clin Exp Allergy 41:29-35

Bu G, Luo Y, Chen F, Liu K, Zhu T (2013) Milk processing as a tool to reduce cow's milk allergenicity: a mini-review. Dairy Sci Technol 93:211-223

Corry DB, Kheradmand F (1999) Induction and regulation of the IgE response. Nature 402:B18-B23

DaVeiga SP (2012) Epidemiology of atopic dermatitis: a review. Allergy Asthma Proc 33:227-234

Iametti S, Transidico P, Bonomi F, Vecchio G, Pittia P, Rovere P, Dall'Aglio G (1997) Molecular modifications of $\beta$-lactoglobulin upon exposure to high pressure. J Agric Food Chem 45:23-29

Isolauri E, Turjanmaa K (1996) Combined skin prick and patch testing enhances identification of food allergy in infants with atopic dermatitis. J Allergy Clin Immunol 97:9-15

Kaczmarski M, Wasilewska J, Cudowska B, Semeniuk J, Klukowski M, Matuszewska E (2013) The natural history of cow's milk allergy in north-eastern Poland. Adv Med Sci 58:22-30

Kang H, Myung EJ, Ahn KS, Eom HJ, Han NS, Kim YB, Kim YJ, Sohn NW (2009) Induction of Th1 cytokines by Leuconostoc mesenteroides subsp. mesenteroides (KCTC 3100) under Th2-type conditions and the requirement of NF-KB and p38/JNK. Cytokine 46:283-289

Kleber N, Maier S, Hinrichs J (2007) Antigenic response of bovine $\beta$-lactoglobulin influenced by ultra-high pressure treatment and temperature. Innov Food Sci Emerg Technol 8:39-45

Leung DY (1999) Pathogenesis of atopic dermatitis. J Allergy Clin Immunol 104:S99-S108

Leung DY (2000) Atopic dermatitis: new insights and opportunities for therapeutic intervention. J Allergy Clin Immunol 105:860-876

Lluis A, Depner M, Gaugler B, Saas P, Casaca VI, Raedler D, Michel S et al (2014) Increased regulatory T-cell numbers are associated with farm milk exposure and lower atopic sensitization and asthma in childhood. $\mathrm{J}$ Allergy Clin Immunol 133:551-559

Matsuda H, Watanabe N, Geba GP, Sperl J, Tsudzuki M, Hiroi J, Matsumoto M, Ushio H, Saito S, Askenase PW, Ra C (1997) Development of atopic dermatitis-like skin lesion with IgE hyperproduction in NC/Nga mice. Int Immunol 461-466

NIAID-Sponsored Expert Panel, Boyce JA, Assa'ad A, Burks AW, Jones SM, Sampson HA, Wood RA, Plaut $\mathrm{M}$ et al (2010) Guidelines for the diagnosis and management of food allergy in the United States: report of the NIAID-sponsored expert panel. J Allergy Clin Immunol 126:S1-S58

Ohmen JD, Hanifin JM, Nickoloff BJ, Rea TH, Wyzykowski R, Kim J, Jullien D, McHugh T, Nassif AS, Chan SC (1995) Overexpression of IL-10 in atopic dermatitis. Contrasting cytokine patterns with delayed-type hypersensitivity reactions. J Immunol 154:1956-1963

Packard KA, Khan MM (2003) Effects of histamine on Th1/Th2 cytokine balance. Int Immunopharmacol 3: 909-920

Roth-Walter F, Berin MC, Arnaboldi P, Escalante CR, Dahan S, Rauch J, Jensen-Jarolim E, Mayer L (2008) Pasteurization of milk proteins promotes allergic sensitization by enhancing uptake through Peyer's patches. Allergy 63:882-890

Sozańska B, Pearce N, Dudek K, Cullinan P (2013) Consumption of unpasteurized milk and its effects on atopy and asthma in children and adult inhabitants in rural Poland. Allergy 68:644-650

Umetsu DT, DeKruyff RH (2006) The regulation of allergy and asthma. Immunol Rev 212:238-255

von Mutius E, Vercelli D (2010) Farm living: effects on childhood asthma and allergy. Nat Rev Immunol 10: 861-868

Won TJ, Kim B, Lim YT, Song DS, Park SY, Park ES, Lee DI, Hwang KW (2011) Oral administration of Lactobacillus strains from Kimchi inhibits atopic dermatitis in NC/Nga mice. J Appl Microbiol 110: 1195-1202 